\title{
Osteometría del Cráneo de la Alpaca Adulta (Vicugna pacos)
}

\author{
Skull Osteometry of the Adult Alpaca (Vicugna pacos) \\ Henry Castañeda C. ${ }^{1}$, Miluska Navarrete Z. ${ }^{1,2}$, Alberto Sato S. ${ }^{\text {, }}$ \\ Alexander Chávez R. ${ }^{1}$
}

\section{Resumen}

El presente estudio tuvo como objetivo la evaluación de la osteometría del cráneo y el cálculo de los índices craneométricos de alpacas (Vicugna pacos) adultas. Se utilizaron 30 cabezas de alpacas Huacaya adultas (15 machos y 15 hembras), provenientes de Sicuani, Cusco, Perú. Los cráneos fueron obtenidos mediante la técnica de maceración. En la descripción anatómica se empleó la terminología recomendada por la Nómina Anatómica Veterinaria 2012. En la medición osteométrica y el cálculo de los índices craneométricos se empleó un vernier digital. El análisis estadístico se hizo con la prueba de «t». Los resultados no indicaron dimorfismo sexual en el aspecto relacionado a la craneometría por efecto del sexo. Los cráneos de la alpaca son de tipo dolicocéfalo con un Índice Cefálico Total de 46.43\%, ultradolicocráneo con un Índice Craneal de 55.42\%. La cara es del tipo hiperlepteno con un Índice Facial de $103.17 \%$, presenta un Índice Mandibular de $62.33 \%$ y el triángulo facial es del tipo escaleno.

Palabras clave: alpaca, cráneo, osteometría, índice craneométrico

\section{AbSTRaCT}

This study aimed to evaluate the skull osteometry and to calculate the craniometric indexes of adult alpacas (Vicugna pacos). The study was carried out using 30 heads of adult Huacaya alpacas (15 males and 15 females), from the district of Sicuani, Cusco, Peru. The skulls were obtained by the maceration technique. The anatomical description was applied using the recommended terminology by the Veterinary Anatomical Nomenclature 2012. A digital vernier was used in the measurement and calculation of craniometric indexes. The «t» test was used to determine differences due to sex. The results showed that adult alpacas do not have sexual dimorphism related to craniometry values. The skulls are the dolichocephalic type with a Total Cephalic Index of $46.43 \%$ and

\footnotetext{
${ }^{1}$ Laboratorio de Anatomía Animal y Fauna Silvestre, Facultad de Medicina Veterinaria, Universidad Nacional Mayor de San Marcos, Lima, Perú

${ }^{2}$ E-mail: miluskabeatriz@gmail.com
}

Recibido: 18 de noviembre de 2015

Aceptado para publicación: 25 de abril de 2016 
ultradolichocranial with a Total Cephalic Index of $55.42 \%$. The alpaca face is the hiperleptene type with a Facial Index of $103.17 \%$, presents a Mandibular Index of $62.33 \%$ and the facial triangle is of scalene type.

Key words: alpaca, skull, osteometry, craniometric index

\section{INTRODUCCIÓN}

El conocimiento actual que se tiene referente a índices osteométricos en camélidos sudamericanos (CSA) es sumamente escaso; más aún en el aspecto relacionado a la craneometría. En el Perú se cuenta con un primer estudio realizado en 1979 (Otte y Venero, 1979), donde se realizaron medidas de cráneos de vicuñas y alpacas pero de manera muy básica y acorde a los conocimientos de esos años. Asimismo, a nivel mundial se han realizado estudios más avanzados y que abarcaron una mayor cantidad de índices osteométricos en especies animales como dromedarios, camellos, ovejas (Özcan et al., 2010; Yahaya et al., 2011, 2012; Louei, 2013b), así como en algunas razas de perros (Louei, 2013a) y animales silvestres como la gacela tibetana (Zhu, 2012).

La literatura científica peruana no muestra estudios adicionales sobre craneometría en CSA hasta el año 2009 (Guillén et al., 2009), donde fue calculado el índice cefálico total de vicuñas machos y hembras adultos vivos, estudio que solo abarcó la medida del largo y ancho de la cabeza.

El Perú es el principal productor de CSA, albergando más del $85 \%$ de alpacas en el mundo (Quispe et al., 2009). Se estima una población nacional de alpacas de 3592 249, siendo los departamentos de Puno, Cusco y Arequipa los mayores productores de alpacas, con el 55,15 y $10 \%$, respectivamente (FAO 2005). Por ello, cobra gran relevancia todo estudio que contribuya a un mejor conocimiento de esta especie animal.
Es así que el conocimiento detallado de los índices craneométricos de la alpaca es importante, no solo en los campos de la comercialización y conservación sino, además, en los campos de la osteoarqueología y zooarqueología, así como para la determinación del dimorfismo sexual, de la anatomía básica, de la morfofisiología de la masticación y en la anestesia regional de la cabeza. No existen reportes completos de la craneometría de la alpaca adulta, de allí que el presente estudio tuvo por objetivo la determinación de las medidas osteométricas y el cálculo de los índices craneométricos.

\section{Materiales y Métodos}

\section{Lugar de Estudio y Animales}

El muestreo del presente estudio se realizó en febrero de 2014 en el camal de Sicuani, localizado en la provincia de Canchis, departamento de Cusco, Perú. Se recolectaron 30 cabezas de alpacas adultas ( 15 hembras y 15 machos), de edades entre 3 y 4 años, de raza Huacaya. Las cabezas fueron trasladadas a la Estación Experimental del Centro de Investigación IVITA-Maranganí, para su acondicionamiento y embalaje y, posteriormente, fueron enviadas al Laboratorio de Anatomía Animal y Fauna Silvestre de la Facultad de Medicina Veterinaria de la Universidad Nacional Mayor de San Marcos, en Lima, para su procesamiento y estudio.

Las cabezas fueron sometidas en Lima a la técnica de maceración con la finalidad de obtener los cráneos. 


\section{TéCniCa de Medición}

La técnica empleada en la medición y cálculo de los índices osteométricos estuvo basada en los estudios hechos por Otte y Venero (1979) y Yahaya et al. (2011, 2012). Las medidas fueron expresadas en centímetros $(\mathrm{cm})$, de acuerdo a los estudios realizados en camellos por Yahaya et al. (2011, 2012) y Louei (2013b), y por Guillén et al. (2009) en vicuñas.

Las medidas e índices osteométricos calculados fueron los siguientes:

\section{Vista dorsal}

- Distancia Interorbital (DIO): Distancia mínima entre los bordes superiores de las órbitas oculares medidas a través de la punta del cráneo (Fig. 1).

- Distancia Intercantal (DIC): Distancia mínima entre los márgenes medios de las órbitas oculares (Fig. 1).

- Ancho del triángulo facial(ATF): La base (en una línea transversal) del triángulo creado por la bifurcación de la cresta sagital externa (Fig. 1).

- Longitud del triángulo facial(LTF): Longitud del lado oblicuo del triángulo creado por la bifurcación de la cresta sagital externa (Fig. 1).

- $\quad$ Altura del triángulo facial(HTF): Altura (en una línea sagital) del triángulo creado por la bifurcación de la cresta sagital externa (Fig. 1).

- $\quad$ Área de un triángulo facial (RTF): (ATF $\mathrm{x} \mathrm{HTF)} / 2$.

- Ancho máximo del neurocráneo (AMN): Distancia máxima entre los extremos laterales de la cavidad craneana (Fig. 2).

- Ancho cigomático máximo (ACM): Distancia máxima entre los arcos cigomáticos (Fig. 2).

- Longitud craneal (LC): Distancia de la línea nucal a la unión de las suturas nasofrontales izquierda y derecha en el plano medio (Fig. 2).
- IÍndice cefálico total (ICT): (AMN x 100) / LC.

- Longitud viscerocraneal (LVC): Distancia de la unión de las suturas nasofrontales izquierda y derecha en el plano medio y el extremo anterior de la punta del cráneo (Fig. 2).

- Índice Facial (IF): (ACM x 100) / LVC.

- Longitud de la cresta sagital externa (LCSE): Distancia desde el extremo caudal de la cresta sagital externa al origen de su bifurcación craneal (Fig. 2).

- Longitud craneal total (LCT): Dimensiones máximas del cráneo cuando se colocan sobre una superficie plana desde la punta craneal de los huesos incisivos hacia el nivel caudal de la línea nucal (Fig. 2).

- Ancho nasal (AN): Ancho máximo de los huesos nasales (Fig. 1).

\section{Vista lateral}

- Altura craneal (sin mandíbula) (AC): Longitud desde punto más alto del hueso frontal a la base del proceso yugular (Fig. 3).

- Diámetro horizontal orbital (DHOB): Diámetro horizontal máximo de la órbita ocular (Fig. 5).

- Diámetro vertical orbital (DVOB): Diámetro vertical máximo de la órbita ocular (Fig. 5).

- $\quad$ Altura del foramen infraorbitario (AFI): Distancia mínima entre los bordes dorsal y ventral del foramen infraorbitario (Fig. 3).

- Foramen infraorbitario a la raíz dorsal de los alvéolos (FIRA): Distancia comprendida desde el borde inferior del foramen infraorbitario directamente a la raíz dorsal de los alvéolos (Fig. 3).

- Foramen infraorbitario a la punta del cráneo (FIPC): Distancia comprendida desde el borde caudal del foramen infraorbitario a la punta del cráneo (Fig. 3).

- Fontanela lagrimal a la punta del cráneo (FLPC): Distancia comprendida desde el borde caudal de la fontanela lagrimal a la punta del cráneo (Fig. 3). 
- Altura maxilar (AM): Distancia comprendida entre el extremo dorsal y ventral del maxilar (Fig. 3).

- Longitud del neurocráneo (LNC): Longitud comprendida a lo largo de la línea media de la indentación más profunda de la unión frontoetmoidal hacia la cara interna de la protuberancia occipital externa (Fig. 4).

- Altura del neurocráneo (HNC): Distancia vertical desde la indentación más profunda de la silla turca hacia la capa interna de la cubierta del cráneo (Fig. 4).

\section{Medidas a nivel mandibular}

- Foramen mentoniano rostral al borde mandibular caudal (FMRBMC): La distancia desde el nivel del foramen mentoniano rostral hasta el extremo del borde caudal de la mandíbula (Fig. 6).

- Foramen mentoniano caudal al borde mandibular caudal (FMCBMC): Distancia desde el nivel del foramen mentoniano caudal al extremo del borde caudal de la mandíbula (Fig. 6).

- Fosa condilar a la base de la mandíbula (FCBM): Distancia de la fosa condilar a la base de mandíbula (Fig. 6).

- Fosa subcondílar a la base de la mandíbula (FSBM): Distancia de la fosa subcondilar a la base de la mandíbula (Fig. 6).

- Longitud mandibular(LM): Longitud de la mandíbula inferior, desde el extremo alveolar hacia el extremo del borde caudal de la mandíbula (Fig. 6).

- Proceso subcondilar a la base de mandíbula (PSBM): Distancia de la proyección máxima del proceso subcondilar a la base de la mandíbula (Fig. 7).

- Altura del cuerpo mandibular en el molar 1 (ACMM1): Altura máxima del cuerpo de la mandíbula a nivel alveolar del molar 1 a la base de la mandíbula (Fig. 7).

- Altura del cuerpo mandibular en el molar 2 (ACMM2): Altura máxima del cuerpo de la mandíbula a nivel alveolar del molar 2 a la base de la mandíbula (Fig. 7).
- $\quad$ Longitud diastemal en adultos (LDA): Longitud de la brecha diastemal, desde el límite caudoventral del primer canino hacia el límite craneoventral del primer premolar (Fig. 6).

- Altura mandibular máxima(AMM): Distancia desde la base de la mandíbula hacia el nivel más alto del proceso coronoides (Fig. 7).

- Foramen mandibular al borde caudal de la mandíbula (FMBC): Longitud desde el extremo del borde caudal de la mandíbula a la línea vertical del foramen mandibular a la base de la mandíbula (Fig. 7).

- Altura máxima del proceso condilar (AMPC): Distancia desde la altura máxima del proceso condilar a la base de la mandíbula (Fig. 7).

- Foramen mandibular a la base de mandíbula (FMBM): Longitud de la línea vertical del límite ventral del foramen mandibular a la base de la mandíbula (Fig. 7).

- Amplitud medial de la cresta mandibular (AMCM): Ancho máximo de la cresta mandibular entre sus puntos más mediales (Fig. 8).

- Longitud mandibular (sínfisis) (LMS): Longitud del límite rostral al límite caudal de la sínfisis mandibular (Fig. 8).

- Amplitud diastemal al foramen mentoniano rostral (ADFMR): Amplitud máxima de la mandíbula en la línea del límite óseo craneal del foramen mentoniano rostral (Fig. 8).

- Amplitud diastemal al foramen mentoniano caudal (ADFMC): Amplitud máxima de la mandíbula en la línea ósea en el extremo caudal del foramen mentoniano caudal (Fig. 8).

- Amplitud condilar 1 (AC1): Amplitud de las mandíbulas entre los puntos más mediales de los procesos condilares derecho e izquierdo (Fig. 9).

- Amplitud condilar 2(AC 2): Amplitud de la mandíbula entre los extremos más laterales de los procesos condilares derecho e izquierdo (Fig. 9). 
- $\quad$ Amplitud intermandibular 1 (AIMB1): Amplitud máxima entre las superficies mediales de las ramas de la mandíbula (Fig. 9).

- Amplitud intermandibular 2(AIMB 2): Amplitud máxima entre las superficies laterales de las ramas de la mandíbula (Fig. 9).

- Espesor mandibular en molar 1 (EMM1): Espesor máximo de la mandíbula en el primer molar (Fig. 8).

- Espesor mandibular en molar2(EMM2): Espesor máximo de la mandíbula en el segundo molar (Fig. 8).

- Espesor mandibular en molar 3(EMM3): Espesor máximo de la mandíbula en el tercer molar (Fig. 8).

\section{Medidas del cráneo con la mandíbula}

- Altura del cráneo completo (ACC): Distancia desde el nivel más alto de la cresta sagital externa hacia la base de la mandíbula (Fig. 5).

- Profundidad mandibular (PM): Distancia medida desde el punto de bifurcación de la cresta sagital externa hacia la base de la mandíbula (Fig. 5).

- Longitud basal (LB): Longitud comprendida desde el extremo alveolar de la mandíbula hacia los cóndilos del occipital a nivel del proceso yugular (Fig. 5).

- Índice mandibular (IM): (PM x 100)/ LB.

- Índice craneal (IC): (AMN x 100)/ LVC.

\section{Vista ventral}

- Amplitud del foramen magno (AFM): Distancia comprendida entre los bordes mediales de los cóndilos occipitales (Fig. 10).

- $\quad$ Ancho máximo de los cóndilos occipitales (ACO): Distancia máxima comprendida entre los bordes laterales de los cóndilos occipitales (Fig. 10).

- Ancho máximo entre los procesos yugulares (APY): Distancia máxima comprendida entre los procesos yugulares (Fig. 10).
- Ancho máximo entre las bullas timpánicas (ABT): Distancia máxima comprendida entre las bullas timpánicas (Fig. 10).

- $\quad$ Longitud del paladar(LP): Longitud del cráneo medida desde la punta del cráneo, determinada por los huesos incisivos, al borde caudal del paladar (Fig. 10).

- $\quad$ Longitud cóndilobasal (LCB): Longitud del cráneo medida desde la punta del cráneo, determinada por los huesos incisivos, a la superficie posterior de los cóndilos del occipital (Fig. 10).

- Longitud de la base del occipital al premolar1 (LBP1): Distancia comprendida entre la porción basilar del occipital al primer premolar (Fig. 10).

- Longitud de la base del occipital al borde caudal del paladar (LBBC): Distancia comprendida entre la porción basilar del occipital al borde caudal del paladar (Fig. 10).

- Distancia del paladar a nivel del molar1 (DPM1): Distancia comprendida entre los bordes mediales del primer molar a nivel del paladar (Fig. 10).

- Distancia del paladar a nivel del molar2 (DPM2): Distancia comprendida entre los bordes mediales del segundo molar a nivel del paladar (Fig. 10).

- Distancia del paladar a nivel del molar3 (DPM3): Distancia comprendida entre los bordes mediales del tercer molar a nivel del paladar (Fig. 10).

Las medidas fueron tomadas tres veces para asegurar la precisión de estas, utilizando como instrumento de medición un vernier digital, con precisión de $\pm 0.05 \mathrm{~mm}$.

\section{Cálculo de los Índices Craneométricos}

a) Índice Cefálico Total (ICT): (ACM x 100) / LCT. De acuerdo al ICT, los tipos de cabeza se clasifican en tres clases: dolicocéfalo (propio del animal de cabeza alargada; ICT es menor de 46\%), mesocéfalo (característico del animal de 


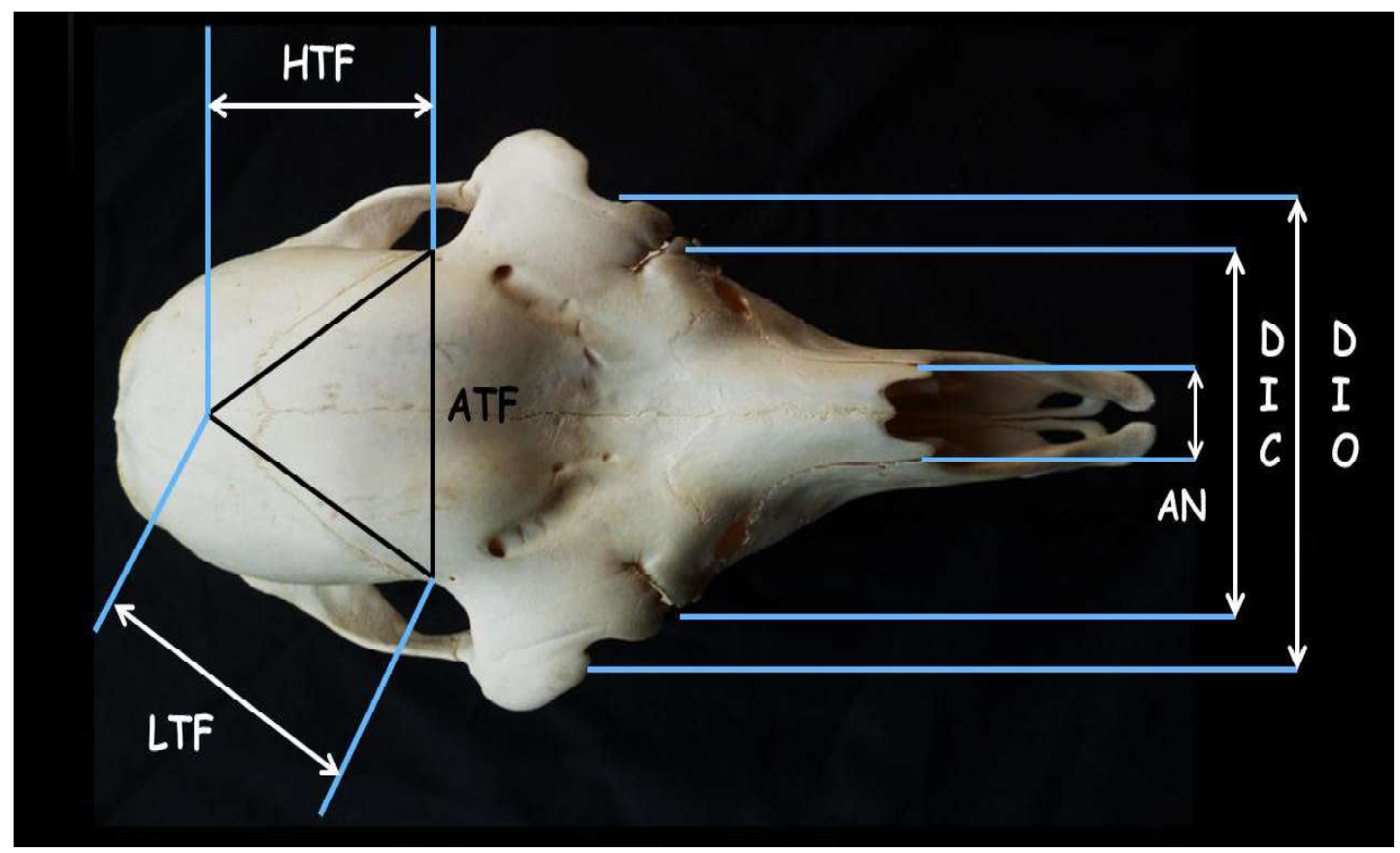

Figura 1. Craneometría. Cráneo de alpaca, vista dorsal. Se muestra Distancia interorbital (DIO); Distancia intercantal (DIC); Ancho del triángulo facial (ATF); Longitud del triángulo facial (LTF); Altura del triángulo facial (HTF); Ancho nasal (AN)

cabeza intermedia; ICT está entre 46 y $55 \%$ ), y braquicéfalo (que define al animal de cabeza corta; ICT es mayor de $55 \%$ ) (Krenzer, 2006; Sañudo, 2009).

b) Índice Facial (IF): (ACM x 100) / LVC. Según el IF, los tipos de cara se clasifican en cuatro clases: hipereurieno (propio del animal de cara muy ancha; IF es menor de $44.9 \%$ ), eurieno (propio del animal de cara ancha: IF entre 45 y $49.9 \%$ ), meseno (IF entre 50 y $54.9 \%$ ), lepteno (propio del animal de cara angosta; IF entre 55 y $59.9 \%$ ) e hiperlepteno (propio del animal de cara muy angosta: IF es mayor de 60\%) (Krenzer, 2006; Sañudo, 2009).

c) Índice Craneal (IC): (AMN x 100) / LVC. De acuerdo al IC, los tipos de cráneo se clasifican en siete clases: ultradolicocráneo (IC es menor de 64.9\%), hiperdolicocráneo (IC entre 65 y 69.9\%), dolicocráneo (IC entre 70 y 74.9\%), mesocráneo (IC entre $75 \mathrm{y}$ $79 \%$ ), braquicráneo (IC entre $80 \mathrm{y}$ $84.9 \%$ ), hiperbraquicráneo (IC entre 85 y $89.9 \%$ ) y ultrabraquicráneo (IC es mayor del 90\%) (Krenzer, 2006; Sañudo, 2009).

d) Índice Mandibular (IM): (PM x 100) / LB

e) Área de un triángulo facial (ATF): (ATF $\mathrm{x} \mathrm{HTF}) / 2$

\section{Análisis Estadístico}

Se determinaron los valores mínimo, máximo, promedio y error estándar de cada medida. Para el análisis de las medidas craneométricas se utilizó el 〈t» test» mediante el programa estadístico MINITAB $(\mathrm{p}<0.05)$ (Özcan et al., 2010) para determinar posibles diferencias estadísticas relacionadas al sexo.

\section{Resultados}

Los valores obtenidos están basados en la totalidad de cráneos (Cuadro 1, 2, 3 y 4) y teniendo en cuenta el sexo de las alpacas. 


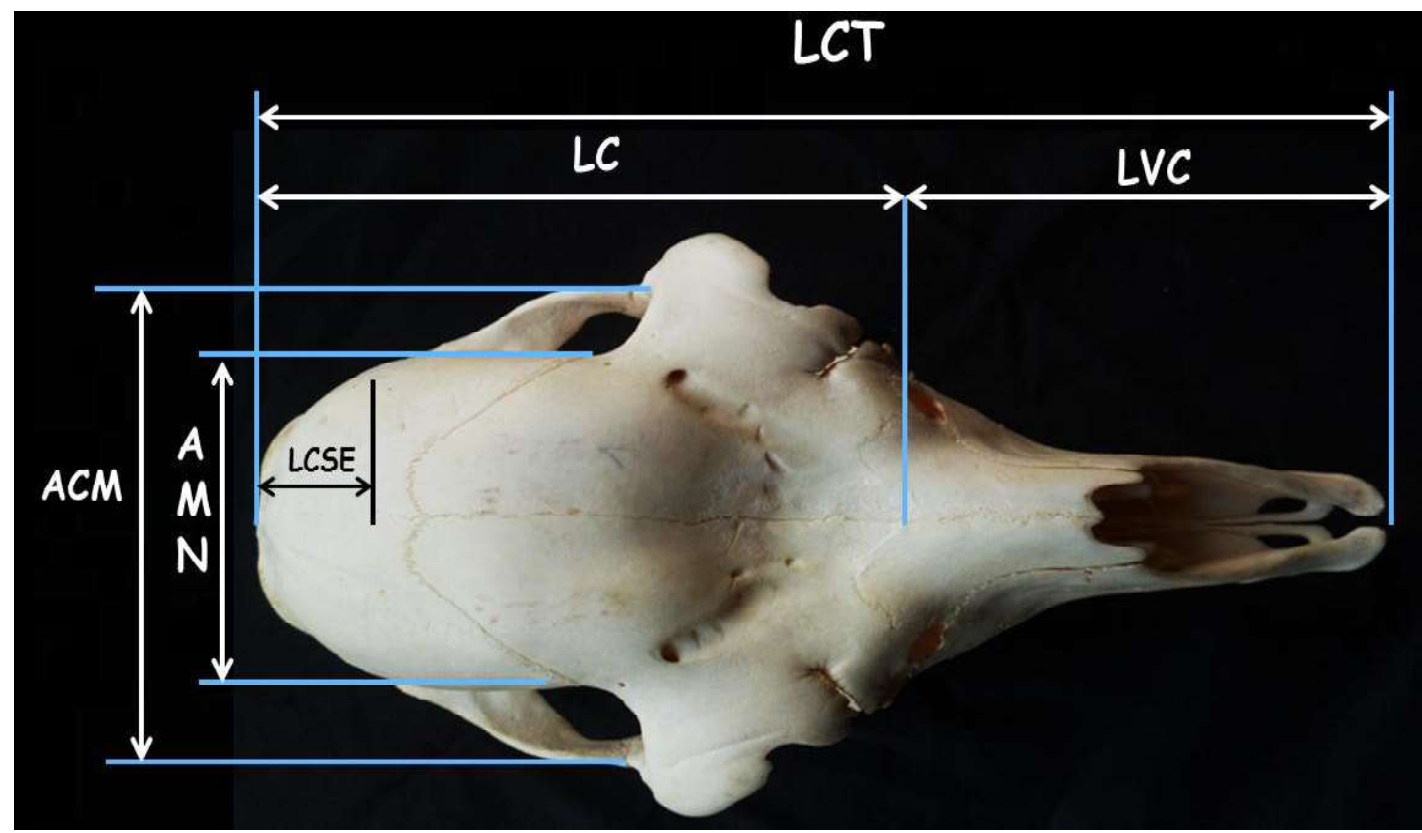

Figura 2. Craneometría. Cráneo de alpaca, vista dorsal. Se muestra Longitud craneal (LC); Ancho cigomático máximo (ACM); Ancho máximo del neurocráneo (AMN); Longitud de la cresta sagital externa (LCSE); Longitud craneal total (LCT); Longitud viscerocraneal (LVC)

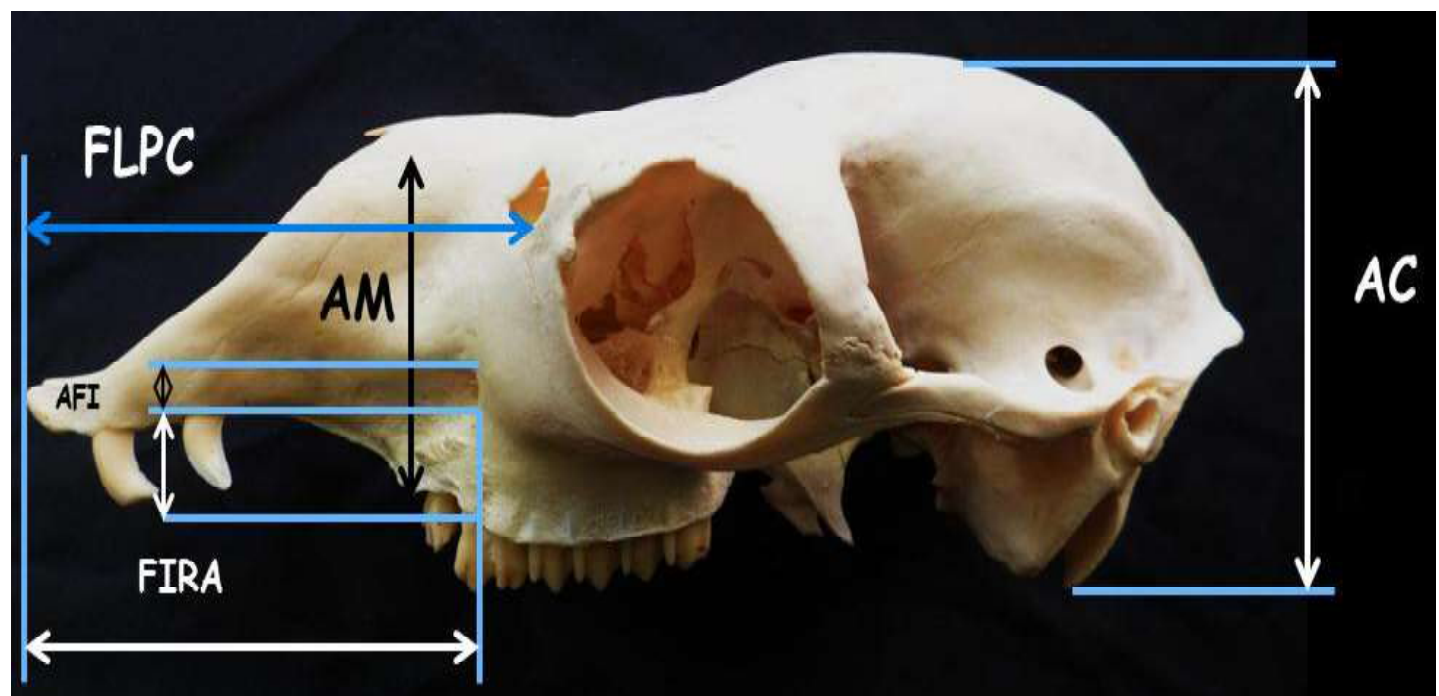

FIPC

Figura 3. Craneometría. Cráneo de alpaca sin mandíbula, vista lateral. Se muestra Altura craneal (sin mandíbula) (AC); Altura del foramen infraorbitario (AFI); Foramen infraorbitario a la raíz dorsal de los alveolos (FIRA); Foramen infraorbitario a la punta del cráneo (FIPC); Fontanela lagrimal a la punta del cráneo (FLPC); Altura maxilar (AM) 


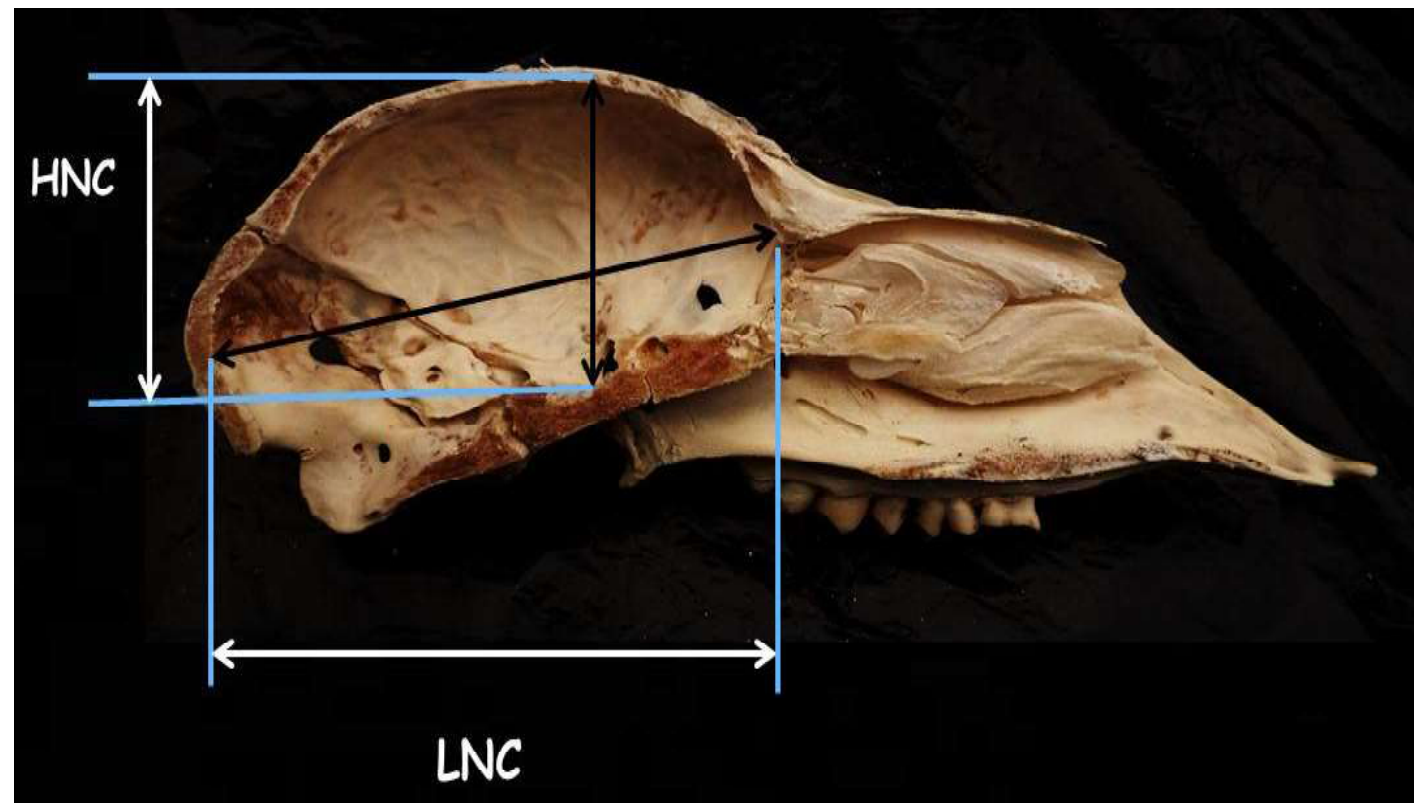

Figura 4. Craneometría. Cráneo de alpaca seccionado sagitalmente. Se muestra Longitud del neurocráneo (LNC); Altura del neurocráneo (HNC)

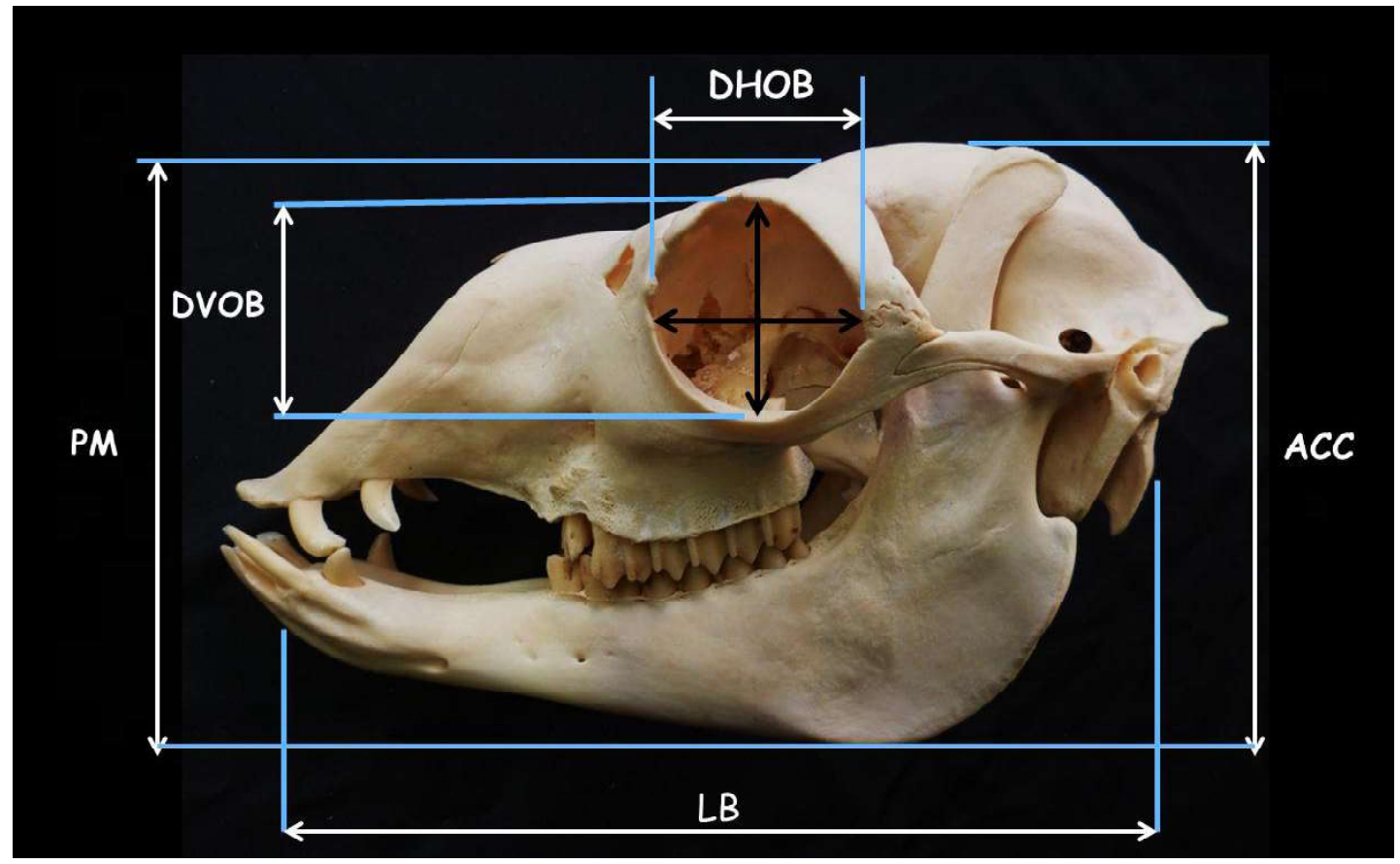

Figura 5. Craneometría. Cráneo de alpaca con la mandíbula. Se muestra Profundidad mandibular (PM); Longitud basal (LB); Altura del cráneo completo (ACC); Diámetro horizontal orbital (DHOB); Diámetro vertical orbital (DVOB) 


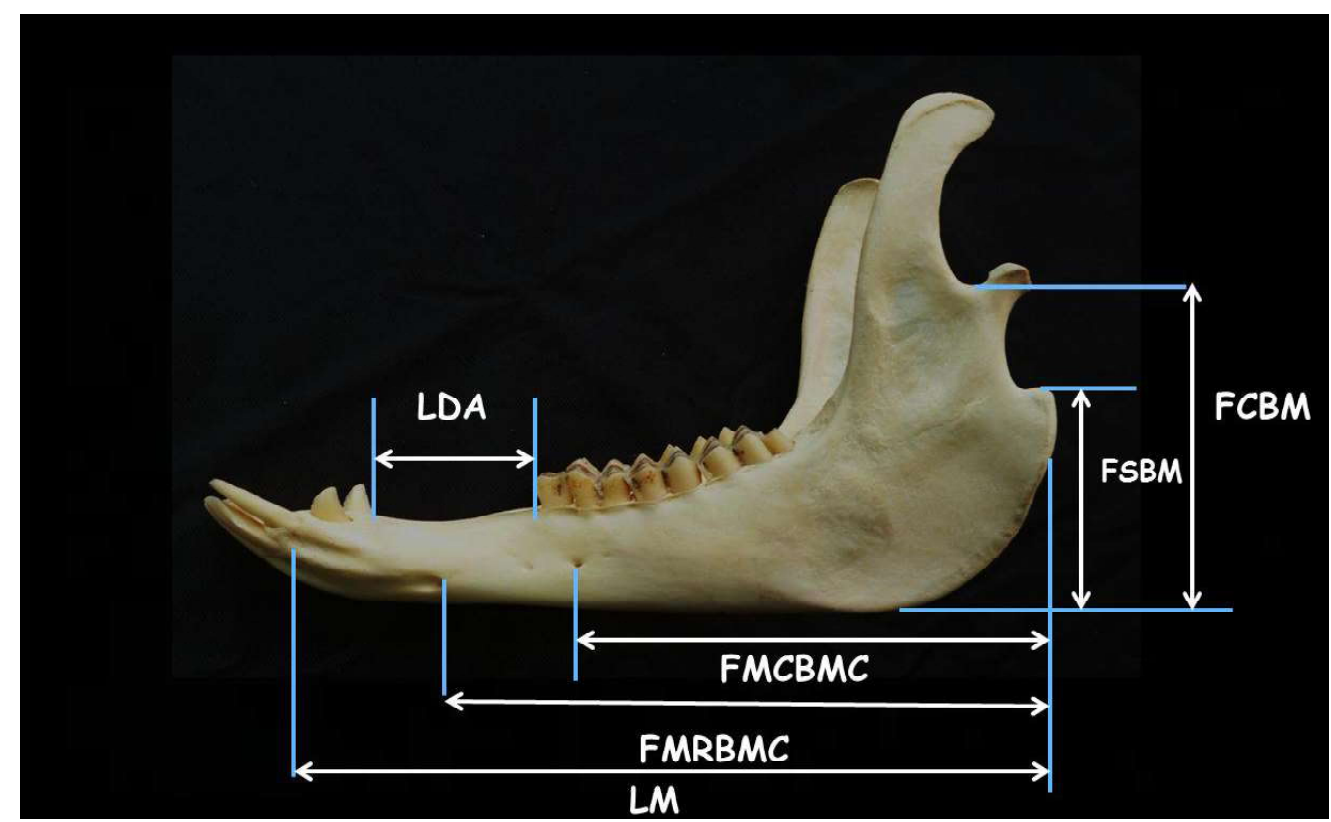

Figura 6. Craneometría. Mandíbula de alpaca, vista lateral. Se muestra Foramen mentoniano rostral al borde mandibular caudal (FMRBMC); Foramen mentoniano caudal al borde mandibular caudal (FMCBMC); Fosa condilar a la base de la mandíbula (FCBM); Fosa subcondilar a la base de la mandíbula (FSBM); Longitud mandibular (LM); Longitud diastemal en adultos (LDA)

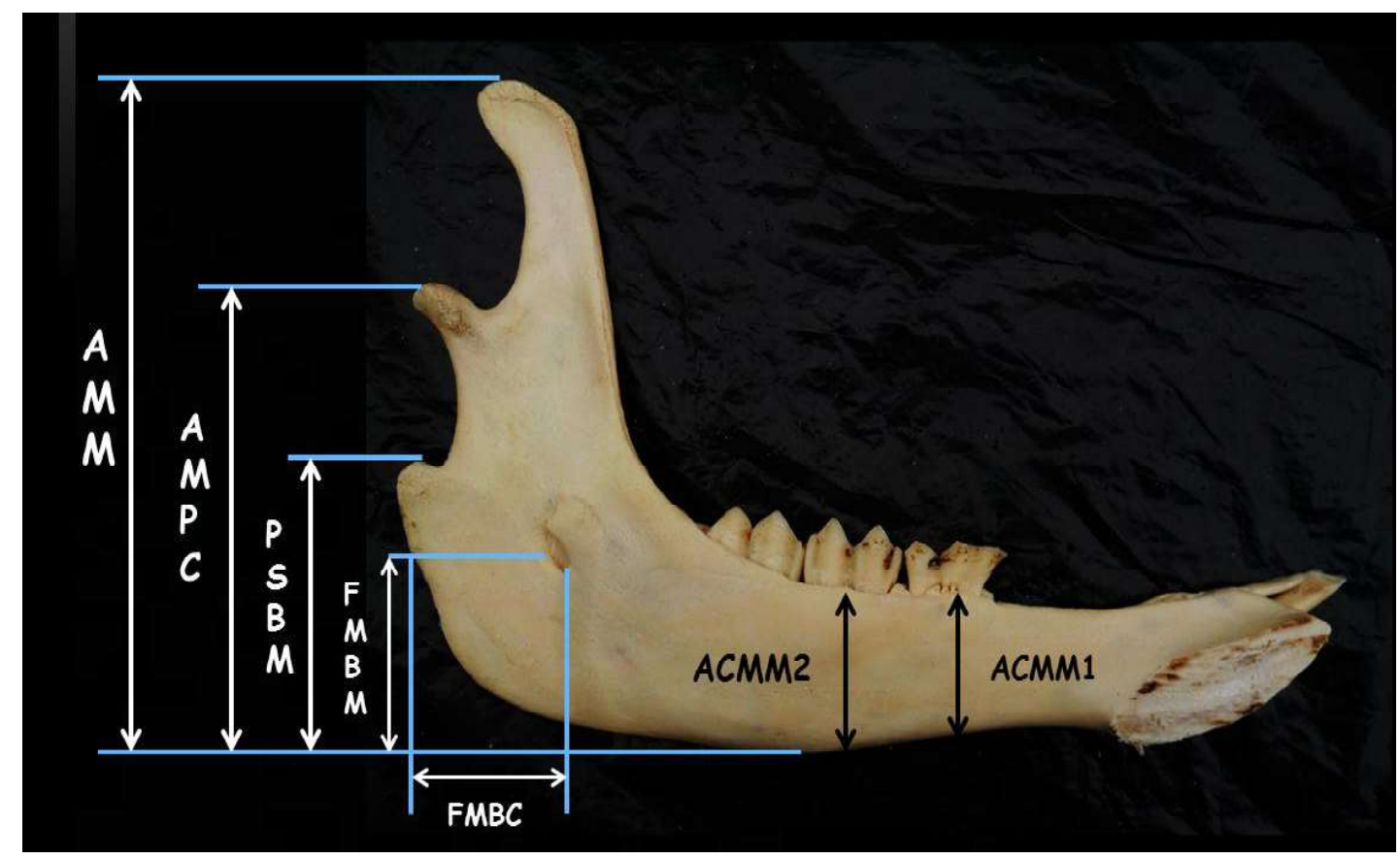

Figura 7. Craneometría. Mandíbula de alpaca, vista medial. Se muestra Altura mandibular máxima (AMM); Altura máxima del proceso condilar (AMPC); Proceso subcondilar a la base de mandíbula (PSBM); Foramen mandibular a la base de mandíbula (FMBM); Foramen mandibular al borde caudal de la mandíbula (FMBC); Altura del cuerpo mandibular en el molar 1 (ACMM1); Altura del cuerpo mandibular en el molar 2 (ACMM2) 


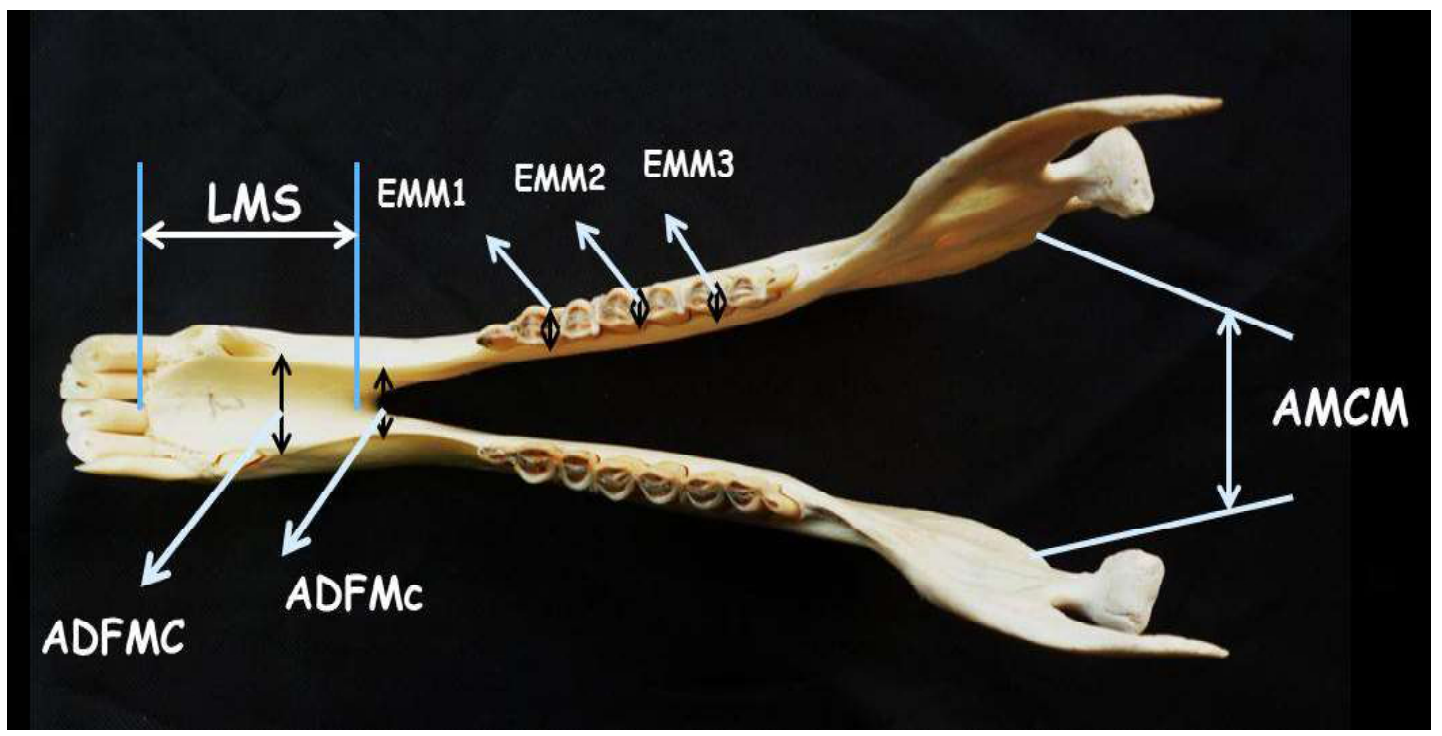

Figura 8. Craneometría. Mandíbula de alpaca, vista dorsal. Se muestra Longitud mandibular (sínfisis) (LMS); Amplitud diastemal al foramen mentoniano rostral (ADFMR); Amplitud diastemal al foramen mentoniano caudal (ADFMC); Amplitud medial de la cresta mandibular (AMCM); Espesor mandibular en molar 1 (EMM1); Espesor mandibular en molar 2 (EMM2); Espesor mandibular en molar 3 (EMM3)

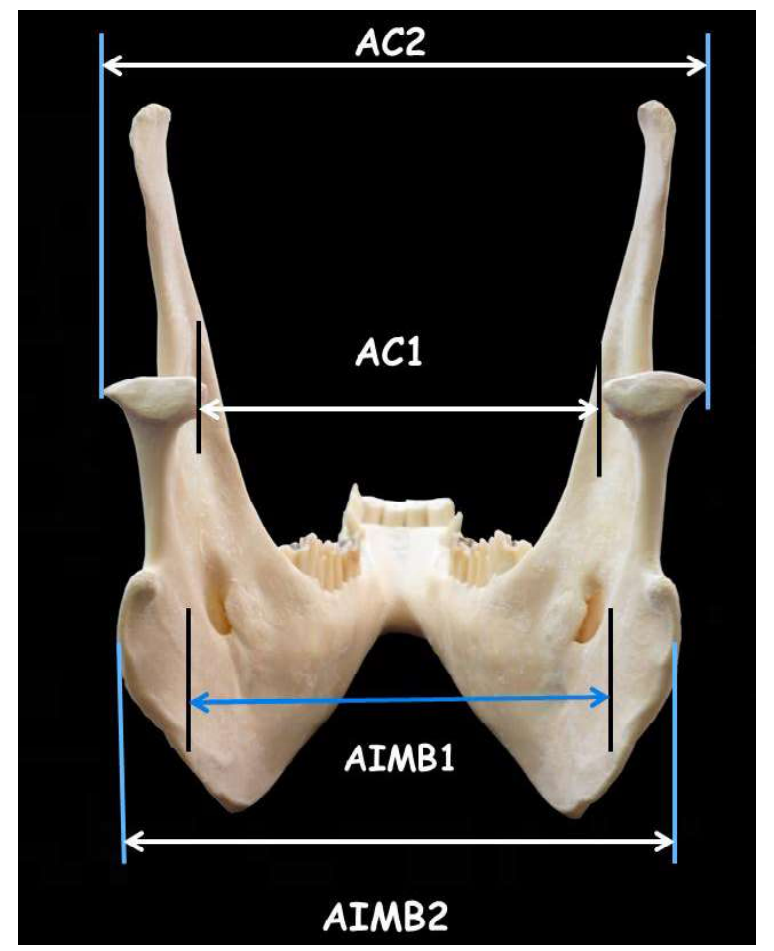

Figura 9. Craneometría. Mandíbula de alpaca, vista caudal. Se muestra Amplitud condilar 1(AC1); Amplitud condilar 2 (AC 2); Amplitud intermandibular 1 (AIMB1); Amplitud intermandibular 2 (AIMB 2) 


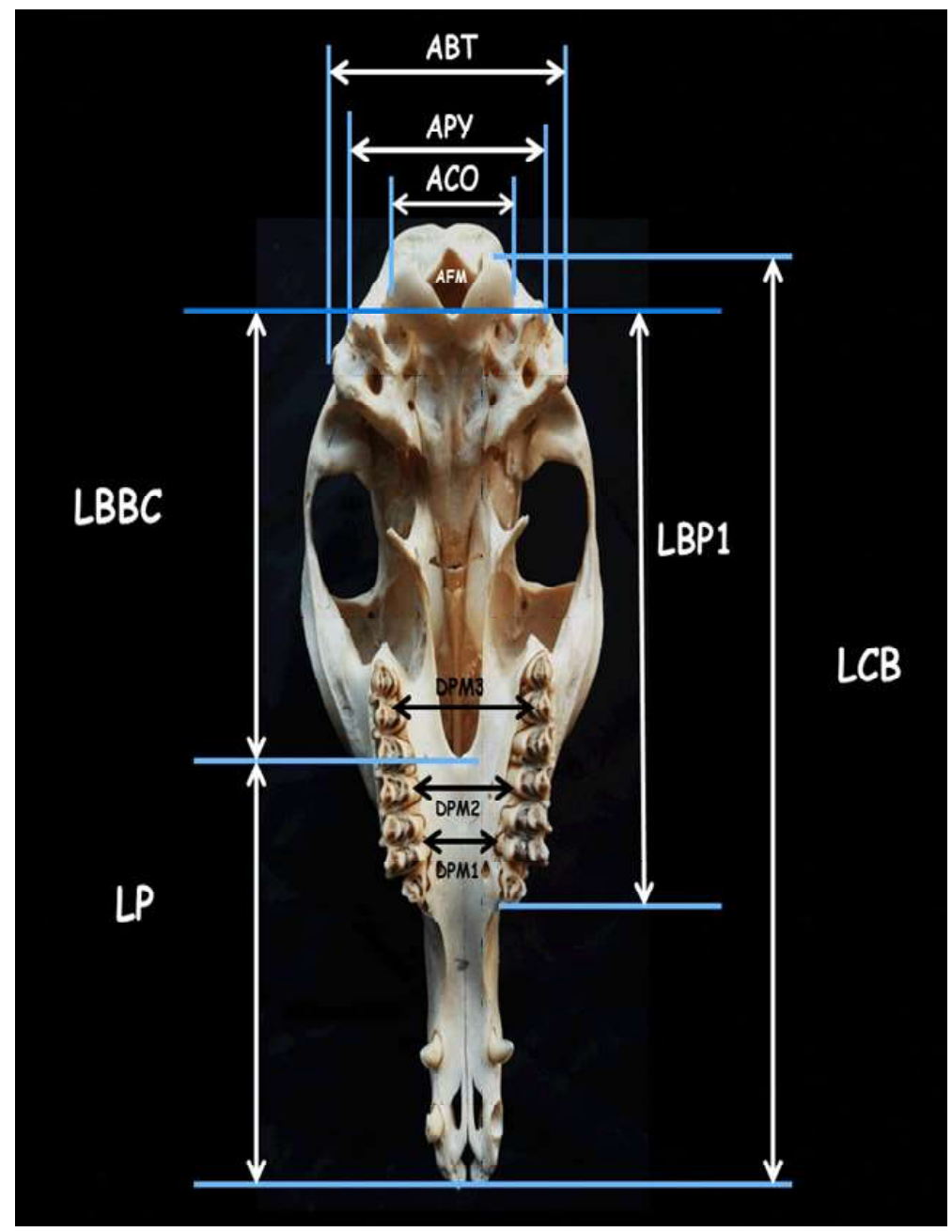

Figura 10. Craneometría. Cráneo de alpaca, vista ventral. Se muestra Amplitud del foramen magno (AFM); Ancho máximo de los cóndilos occipitales (ACO); Ancho máximo entre los procesos yugulares (APY); Ancho máximo entre las bullas timpánicas (ABT); Longitud del paladar (LP); Longitud condilobasal (LCB); Longitud de la base del occipital al premolar 1 (LBP1); Longitud de la base del occipital al borde caudal del paladar (LBBC); Distancia del paladar a nivel del molar 1 (DPM1); Distancia del paladar a nivel del molar 2 (DPM2); Distancia del paladar a nivel del molar 3 (DPM3).

Los índices craneométricos de la alpaca adulta fueron:

- Índice Cefálico Total (ICT): (11.9 x 100)/ $25.63=46.43$ o $46.4 \%$

- Índice Facial (IF): $(11.9 \times 100) / 11.35=$ 104.84 o $104.8 \%$
- Índice Craneal (IC): $(6.29 \times 100) / 11.35=$ 55.42 o $55.4 \%$

- Índice Mandibular (IM): (13.83 x 100) / $22.19=62.33$ o $62.3 \%$

- Área de un triángulo facial (ATF): (5.89 x 5.08) $/ 2=14.96$ 
Cuadro 1. Medidas osteométricas $(\mathrm{cm})$ del cráneo $(\mathrm{n}=30)$ de la alpaca adulta (Vicugna pacos). Parte 1

\begin{tabular}{|c|c|c|c|c|c|c|c|c|}
\hline Índices & Mt & Vmt & $\bar{X} M$ & $\overline{\mathrm{X}} \mathrm{H}$ & $\sigma \mathrm{M}$ & $\sigma \mathrm{H}$ & $\overline{\mathrm{X}} \mathrm{t}$ & $\sigma \tau$ \\
\hline \multicolumn{9}{|l|}{ Vista dorsal } \\
\hline Distancia inte & 0.4 & 9.55 & 9.89 & 9.96 & 0.28 & 0.29 & 9.93 & 0.29 \\
\hline Distal & .86 & 7.62 & 7.97 & 8.13 & 0.25 & 0.36 & 8.05 & 0.31 \\
\hline Ancho del triángulo facial (ATF) & 6.3 & 5.22 & 5.82 & 5.97 & 0.36 & 0.27 & 5.89 & 0.32 \\
\hline Longitud del triángulo facial (LTF) & 5.84 & 4.71 & 5.31 & 5.47 & 0.34 & 0.27 & 5.39 & 0.32 \\
\hline Altura del triángulo facial (HTF) & 5.54 & 4.43 & 5.01 & 5.16 & 0.36 & 0.26 & 5.08 & 0.32 \\
\hline $\begin{array}{l}\text { Ancho máximo del neurocráneo } \\
\text { (AMN) }\end{array}$ & 78 & 42 & 6.31 & 6.28 & 0.39 & 0.35 & 6.29 & 36 \\
\hline Ancho cigomático máximo (ACN & & 10.76 & 11.78 & 12.02 & 0.66 & 0.51 & 11.9 & 0.59 \\
\hline Long & 3 & 86 & 14.29 & 14.4 & 0.2 & 0.37 & 14.4 & 0.33 \\
\hline Longit & 11.99 & 10.45 & 11.19 & 11.5 & 0.42 & 0.37 & 11.4 & 0.42 \\
\hline $\begin{array}{l}\text { Long } \\
\text { (LCS }\end{array}$ & 4.42 & 3.36 & 4.04 & 4.2 & 0.3 & 0.15 & 4.12 & 0.25 \\
\hline craneal total (LCT) & 26.98 & 23.65 & 25.49 & 25.77 & 0.63 & 0.87 & 25.6 & 0.76 \\
\hline Ancho nasal (AN) & 2.31 & 2.1 & 2.18 & 2.23 & 0.06 & 0.05 & 2.2 & 0.06 \\
\hline \multicolumn{9}{|l|}{ Vista lateral } \\
\hline & & & 8.17 & 8.36 & 0.22 & 0.35 & 8.47 & 0.31 \\
\hline Diámetro hor & 4.46 & 2.25 & 3.82 & 4.05 & 0.59 & 0.6 & 3.94 & 0.6 \\
\hline Diámetro vertical orbital (DVOB) & 4.41 & 2.18 & 3.76 & 3.87 & 0.55 & 0.55 & 3.81 & 0.54 \\
\hline $\begin{array}{l}\text { Altura } \\
\text { (AFI) }\end{array}$ & .89 & 0.51 & 0.67 & 0.79 & 0.11 & 0.11 & 0.73 & 0.13 \\
\hline $\begin{array}{l}\text { Foramen infraorbitario a la raíz } \\
\text { dorsal de los alveolos (FIRA) }\end{array}$ & דט. & 1.21 & 1.38 & 1.44 & 0.09 & 0.08 & 1.41 & 0.09 \\
\hline punta del & 11.0 & 8.08 & 9.17 & 9.72 & 0.78 & 0.69 & 9.44 & 0.78 \\
\hline $\begin{array}{l}\text { Fontanela lagrimal a la punta del } \\
\text { cráneo (FLPC) }\end{array}$ & 12.0 & 10.4 & 11.4 & 11.3 & 0.2 & 0.33 & 11.4 & 0.28 \\
\hline Altura maxilar (AM) & 5.46 & 4.68 & 4.91 & 5.04 & 0.15 & 0.23 & 4.98 & 0.21 \\
\hline Altura del neurocráneo (HNC) & 6.2 & 5.19 & 5.58 & 5.43 & 0.23 & 0.2 & 5.5 & 0.23 \\
\hline Longitud del neurocráneo (LNC) & 10.0 & 8.56 & 9.2 & 9.25 & 0.35 & 0.25 & 9.23 & 0.29 \\
\hline
\end{tabular}

VMt: Valor máximo del total de cráneos; Vmt: Valor mínimo del total de cráneos; $0 \mathrm{M}$ : Promedio en machos; $\sigma \mathrm{M}$ : Desviación estándar en machos; $\mathrm{OH}$ : Promedio en hembras; $\sigma \mathrm{H}$ : Desviación estándar en hembras; 0 t: Promedio total; $\sigma$ t: Desviación estándar total 
Cuadro 2. Medidas osteométricas $(\mathrm{cm})$ del cráneo - Nivel mandibular $(\mathrm{n}=30)$ de la alpaca adulta (Vicugna pacos). Parte 2

\begin{tabular}{|c|c|c|c|c|c|c|c|c|}
\hline $\begin{array}{l}\text { Medidas a nivel } \\
\text { mandibular }\end{array}$ & VMt & Vmt & $\bar{X} M$ & $\overline{\mathrm{X}} \mathrm{H}$ & $\sigma \mathrm{M}$ & $\sigma \mathrm{H}$ & $\bar{x} t$ & $\sigma \tau$ \\
\hline $\begin{array}{l}\text { Foramen mentoniano rostral } \\
\text { al borde mandibular caudal } \\
\text { (FMRBMC) }\end{array}$ & 15.31 & 13.66 & 14.74 & 14.79 & 0.49 & 0.49 & 14.77 & 0.48 \\
\hline $\begin{array}{l}\text { Foramen mentoniano caudal } \\
\text { al borde mandibular caudal } \\
\text { (FMCBMC) }\end{array}$ & 11.86 & 10.02 & 11.04 & 10.98 & 0.44 & 0.39 & 11.01 & 0.41 \\
\hline $\begin{array}{l}\text { Fosa condílea a la base de la } \\
\text { mandíbula (FCBM) }\end{array}$ & 13.92 & 12.11 & 12.96 & 12.75 & 0.49 & 0.41 & 12.85 & 0.45 \\
\hline $\begin{array}{l}\text { Fosa subcondílea a la base } \\
\text { de la mandíbula (FSBM) }\end{array}$ & 11.4 & 10.36 & 11.02 & 10.75 & 0.2 & 0.29 & 10.89 & 0.28 \\
\hline Longitud mandibular (LM) & 18.9 & 17.33 & 17.93 & 18.18 & 0.32 & 0.45 & 18.06 & 0.39 \\
\hline $\begin{array}{l}\text { Proceso subcondilar a la } \\
\text { base de mandíbula (PSBM) }\end{array}$ & 6.7 & 4.82 & 5.61 & 6.19 & 0.57 & 0.49 & 5.89 & 0.59 \\
\hline $\begin{array}{l}\text { Altura del cuerpo } \\
\text { mandibular en el molar } 1 \\
\text { (ACMM1) }\end{array}$ & 2.89 & 2.23 & 2.67 & 2.63 & 0.06 & 0.15 & 2.65 & 0.11 \\
\hline $\begin{array}{l}\text { Altura del cuerpo } \\
\text { mandibular en el molar } 2 \\
\text { (ACMM2) }\end{array}$ & 3.25 & 2.73 & 3.13 & 3.09 & 0.08 & 0.15 & 3.11 & 0.12 \\
\hline $\begin{array}{l}\text { Longitud diastemal en } \\
\text { adultos (LDA) }\end{array}$ & 4.9 & 3.45 & 4.0 & 4.23 & 0.41 & 0.41 & 4.11 & 0.42 \\
\hline $\begin{array}{l}\text { Altura mandibular máxima } \\
\text { (AMM) }\end{array}$ & 13.91 & 12.18 & 12.95 & 13.22 & 0.53 & 0.53 & 13.09 & 0.54 \\
\hline $\begin{array}{l}\text { Foramen mandibular al } \\
\text { borde caudal de la } \\
\text { mandíbula (FMBC) }\end{array}$ & 3.07 & 2.35 & 2.59 & 2.73 & 0.19 & 0.21 & 2.66 & 0.21 \\
\hline $\begin{array}{l}\text { Altura máxima del proceso } \\
\text { condilar (AMPC) }\end{array}$ & 10.01 & 8.52 & 9.13 & 9.46 & 0.47 & 0.49 & 9.29 & 0.5 \\
\hline $\begin{array}{l}\text { Foramen mandibular a la } \\
\text { base de mandíbula (FMBM) }\end{array}$ & 4.96 & 3.32 & 3.84 & 4.07 & 0.29 & 0.41 & 3.96 & 0.37 \\
\hline $\begin{array}{l}\text { Amplitud medial de la } \\
\text { cresta mandibular (AMCM) }\end{array}$ & 7.08 & 5.69 & 6.57 & 6.83 & 0.39 & 0.33 & 6.69 & 0.38 \\
\hline
\end{tabular}

VMt: Valor máximo del total de cráneos; Vmt: Valor mínimo del total de cráneos; OM: Promedio en machos; $\sigma \mathrm{M}$ : Desviación estándar en machos; $\mathrm{OH}$ : Promedio en hembras; $\sigma \mathrm{H}$ : Desviación estándar en hembras; 0t: Promedio total; $\sigma \mathrm{t}$ : Desviación estándar total 
Cuadro 3. Medidas osteométricas $(\mathrm{cm})$ del cráneo - Nivel mandibular $(\mathrm{n}=30)$ de la alpaca adulta (Vicugna pacos). Parte 3

\begin{tabular}{|c|c|c|c|c|c|c|c|c|}
\hline Medidas a nivel mandibular & VMt & Vmt & $\bar{X} M$ & $\overline{\mathrm{X}} \mathrm{H}$ & $\sigma \mathrm{M}$ & $\sigma \mathrm{H}$ & $\overline{\mathrm{X}} \mathrm{t}$ & $\sigma \tau$ \\
\hline $\begin{array}{l}\text { Longitud mandibular } \\
\text { (sínfisis) (LMS) }\end{array}$ & 5.26 & 4.5 & 4.84 & 4.88 & 0.25 & 0.25 & 4.86 & 0.25 \\
\hline $\begin{array}{l}\text { Amplitud diastemal al } \\
\text { foramen mentoniano rostral } \\
\text { (ADFMR) }\end{array}$ & 1.87 & 1.01 & 1.29 & 1.22 & 0.3 & 0.18 & 1.26 & 0.25 \\
\hline $\begin{array}{l}\text { Amplitud diastemal al } \\
\text { foramen mentoniano caudal } \\
\text { (ADFMC) }\end{array}$ & 1.22 & 0.83 & 0.9 & 0.95 & 0.09 & 0.09 & 0.93 & 0.09 \\
\hline Amplitud condilar 1 (AC1) & 5.94 & 4.93 & 5.51 & 5.48 & 0.25 & 0.22 & 5.49 & 0.23 \\
\hline Amplitud condilar 2(AC 2) & 9.34 & 7.94 & 8.45 & 8.73 & 0.37 & 0.43 & 8.59 & 0.42 \\
\hline $\begin{array}{l}\text { Amplitud intermandibular } 1 \\
\text { (AIMB1) }\end{array}$ & 7.58 & 5.52 & 6.38 & 6.69 & 0.48 & 0.51 & 6.54 & 0.51 \\
\hline $\begin{array}{l}\text { Amplitud intermandibular } 2 \\
\text { (AIMB2) }\end{array}$ & 8.3 & 7 & 7.45 & 7.57 & 0.33 & 0.36 & 7.51 & 0.35 \\
\hline $\begin{array}{l}\text { Espesor mandibular en molar } \\
1 \text { (EMM1) }\end{array}$ & 0.92 & 0.6 & 0.68 & 0.75 & 0.08 & 0.11 & 0.72 & 0.1 \\
\hline $\begin{array}{l}\text { Espesor mandibular en molar } \\
2 \text { (EMM2) }\end{array}$ & 1.21 & 0.92 & 0.99 & 1.04 & 0.07 & 0.09 & 1.02 & 0.09 \\
\hline $\begin{array}{l}\text { Espesor mandibular en molar } \\
3 \text { (EMM3) }\end{array}$ & 1.42 & 1.18 & 1.26 & 1.29 & 0.05 & 0.07 & 1.27 & 0.06 \\
\hline \multicolumn{9}{|l|}{$\begin{array}{l}\text { Medidas del cráneo con la } \\
\text { mandíbula }\end{array}$} \\
\hline $\begin{array}{l}\text { Altura del cráneo completo } \\
\text { (ACC) }\end{array}$ & 14.85 & 13.25 & 13.82 & 14.33 & 0.52 & 0.41 & 14.08 & 0.53 \\
\hline $\begin{array}{l}\text { Profundidad mandibular } \\
\text { (PM) }\end{array}$ & 14.55 & 13.04 & 13.69 & 13.98 & 0.44 & 0.36 & 13.83 & 0.42 \\
\hline Longitud basal (LB) & 22.89 & 21.33 & 22.04 & 22.34 & 0.43 & 0.39 & 22.19 & 0.43 \\
\hline
\end{tabular}

VMt: Valor máximo del total de cráneos; Vmt: Valor mínimo del total de cráneos; OM: Promedio en machos; $\sigma \mathrm{M}$ : Desviación estándar en machos; $\mathrm{OH}$ : Promedio en hembras; $\sigma \mathrm{H}$ : Desviación estándar en hembras; 0 t: Promedio total; $\sigma \mathrm{t}$ : Desviación estándar total

\section{Discusión}

En la vista lateral del cráneo se encuentra la fontanela lagrimal, que es un foramen delimitado por el hueso frontal, el hueso nasal, el hueso lagrimal y el hueso maxilar. Este foramen tiene comunicación directa con el seno frontal en la parte dorsal ya que los divi- de un tabique incompleto en la parte ventral (Zárate, 2014). Antiguamente se le denominaba foramina infraorbitaria (Otte y Venero, 1979) y fue ilustrado por primera vez por Galotta y Galota (1988) en un atlas de osteología de la llama. Las fontanelas están ausentes en el caso de las vicuñas, habiendo en su lugar una articulación fronto-maxilar (Pacheco et al., 1979). 
Cuadro 4. Medidas osteométricas $(\mathrm{cm})$ del cráneo $(\mathrm{n}=30)$ de la alpaca adulta (Vicugna pacos). Parte 4

\begin{tabular}{|c|c|c|c|c|c|c|c|c|}
\hline Vista ventral & VMt & Vmt & $\bar{X} M$ & $\overline{\mathrm{X}} \mathrm{H}$ & $\sigma \mathrm{M}$ & $\sigma \mathrm{H}$ & $\overline{\mathrm{X}} \mathrm{t}$ & $\sigma \tau$ \\
\hline $\begin{array}{l}\text { Amplitud del foramen magno } \\
\text { (AFM) }\end{array}$ & 2.14 & 1.35 & 1.89 & 1.93 & 0.17 & 0.18 & 1.91 & 0.17 \\
\hline $\begin{array}{l}\text { Ancho máximo de los } \\
\text { cóndilos occipitales (ACO) }\end{array}$ & 4.44 & 3.14 & 3.83 & 3.81 & 0.19 & 0.3 & 3.82 & 0.25 \\
\hline $\begin{array}{l}\text { Ancho máximo entre los } \\
\text { procesos yugulares (APY) }\end{array}$ & 6.6 & 5.26 & 5.95 & 5.97 & 0.44 & 0.38 & 5.96 & 0.4 \\
\hline $\begin{array}{l}\text { Ancho máximo entre las } \\
\text { bullas timpánicas (ABT) }\end{array}$ & 7.89 & 6.82 & 7.27 & 7.33 & 0.29 & 0.27 & 7.3 & 0.28 \\
\hline Longitud del paladar (LP) & 12.46 & 10.14 & 10.87 & 11.79 & 0.79 & 0.76 & 11.33 & 0.89 \\
\hline Longitud cóndilobasal (LCB) & 23.51 & 20.37 & 21.7 & 22.23 & 0.9 & 0.72 & 21.97 & 0.85 \\
\hline $\begin{array}{l}\text { Longitud de la base del } \\
\text { occipital al premolar } 1 \\
\text { (LBP1) }\end{array}$ & 13.89 & 11.94 & 12.59 & 12.84 & 0.49 & 0.49 & 12.71 & 0.49 \\
\hline $\begin{array}{l}\text { Longitud de la base del } \\
\text { occipital al borde caudal del } \\
\text { paladar (LBBC) }\end{array}$ & 9.82 & 8.68 & 9.34 & 9.41 & 0.43 & 0.33 & 9.38 & 0.38 \\
\hline $\begin{array}{l}\text { Distancia del paladar a nivel } \\
\text { del molar } 1 \text { (DPM1) }\end{array}$ & 3.3 & 2.24 & 2.75 & 2.96 & 0.31 & 0.3 & 2.85 & 0.32 \\
\hline $\begin{array}{l}\text { Distancia del paladar a nivel } \\
\text { del molar } 2 \text { (DPM2) }\end{array}$ & 4.12 & 3.3 & 3.69 & 3.87 & 0.32 & 0.24 & 3.78 & 0.29 \\
\hline $\begin{array}{l}\text { Distancia del paladar a nivel } \\
\text { del molar } 3 \text { (DPM3) }\end{array}$ & 4.61 & 3.85 & 4.28 & 4.38 & 0.22 & 0.22 & 4.33 & 0.22 \\
\hline
\end{tabular}

VMt: Valor máximo del total de cráneos; Vmt: Valor mínimo del total de cráneos; 0 M: Promedio en machos; $\sigma \mathrm{M}$ : Desviación estándar en machos; $\mathrm{OH}$ : Promedio en hembras; $\sigma \mathrm{H}$ : Desviación estándar en hembras; 0t: Promedio total; $\sigma \mathrm{t}$ : Desviación estándar total

A nivel del temporal, la bulla timpánica en la alpaca y en el camello es una lámina ósea comprimida lateralmente, mientras que en la llama es proporcionalmente más grande y redondeada (Fuentes, 1953). En el caso de la vicuña presenta algunas perforaciones y una forma que lo asemeja a un panal de abejas. Asimismo, en el bovino es larga, comprimida lateralmente y proporcionalmente más grande que en la llama y no existe en el equino (Sisson y Grossman, 2000).
A nivel mandibular, el cráneo de alpaca presenta tres apófisis, siendo el proceso coronoides, el proceso condilar y una apófisis pequeña que sigue al proceso condilar denominado proceso subcondilar, característico de esta especie (Navarrete y Sato, 2010). Antiguamente se denominaba proceso angular por extrapolación del proceso angular en los caninos; asimismo, en el caso de los camellos esta tercera apófisis se denomina proceso angular (Yahaya et al., 2011). En la superficie lateral del cuerpo y a nivel de la raíz 
del primer molar, la alpaca y la llama presentan el foramen mentoniano accesorio, donde este agujero es denominado foramen anterior dental inferior en el camello (Fuentes, 1953), mientras que no se observa en el bovino y el equino (Sisson y Grossman, 2000).

A nivel del occipital, en la cara ventral de la alpaca se observa dos pequeños tubérculos musculares poco desarrollados al igual que la llama y el equino, mientras que en el bovino son bien manifiestos (Sisson y Grossman, 2000).

Cabe recalcar que la NAV (ICVGAN, 2012) no hace referencia específica a los CSA, al igual que los camélidos del viejo mundo, pues estos se circunscriben a regiones específicas del mundo; así tenemos por ejemplo, la fontanela lagrimal, el proceso subcondíleo y la fosa subcondilar, que son estructuras exclusivas de los camélidos. Otras estructuras anatómicas se describen haciendo una extrapolación de especies, como el equino y el bovino.

Los estudios más recientes relacionados a la osteometría de cráneo en alpacas corresponden a los realizados por Otte y Venero (1979), donde se utilizaron 25 cráneos de alpacas adultas. Allí se registraron 43 mediciones por cráneo (30 en la parte superior y 13 en la mandíbula); sin embargo, los términos anatómicos utilizados no están adecuados a la NAV (ICVGAN, 2012), sino que son una adaptación de términos anatómicos utilizados en los tratados de anatomía forense humana. Asimismo, no se indica la edad ni la raza de las alpacas estudiadas. Por tanto, en el presente trabajo se tomaron como base no solo esos resultados sino principalmente los estudios realizados por Yahaya et al. (2011, 2012) en dromedarios, pues no solo presentan un protocolo de mediciones más completo sino que hacen uso de la NAV (ICVGAN, 2012) en la identificación de las estructuras anatómicas, realizándose 64 mediciones craneométricas (40 en la parte superior y 20 en la mandíbula) y el cálculo de cinco índices craneométricos.
Entre los resultados más relevantes se pueden considerar los siguientes:

- La longitud condilobasal tuvo un valor promedio de $23.23 \mathrm{~cm}$ en el estudio de Otte y Venero (1979); sin embargo, en el presente estudio fue de $21.97 \mathrm{~cm}$ y un valor máximo de $23.51 \mathrm{~cm}$ en una hembra adulta. Asimismo, Otte y Venero (1979) indican una altura maxilar de 5.5 $\mathrm{cm}$ y en este caso fue de $4.98 \mathrm{~cm}$. Estas diferencias podrían deberse al efecto raza y sexo, pues dichos autores no indicaron la raza ni el número de animales por sexo.

- La longitud total del cráneo fue de 24.87 $\mathrm{cm}$, en tanto que en el estudio biométrico de Romero (1989) en alpacas Huacaya adultas fue de $25.63 \mathrm{~cm}$, pero cabe recalcar que dicha medición se realizó en alpacas vivas. Asimismo, Yahaya et al. (2011) establecieron como límite posterior de la longitud craneal total en los dromedarios a la cresta nucal; estructura propia de los carnívoros, conejos, caballos y cerdos. En el presente trabajo se estableció como límite posterior a la línea nucal, pues las alpacas al igual que los rumiantes no presentan una cresta propiamente dicha a este nivel.

- La relación promedio entre largo y altura del cráneo en la alpaca fue de $1: 3.0 \mathrm{y}$ entre el largo y el ancho del cráneo fue de 1:4 (Otte y Venero, 1979), las cuales se corresponden a los resultados de este estudio.

- $\quad$ El ancho cigomático máximo fue de 11.5 cm (Otte y Venero, 1979), el cual se corresponde al valor obtenido de $11.35 \mathrm{~cm}$. Asimismo, el valor hallado por Romero (1989) fue de $11.81 \mathrm{~cm}$.

- La longitud del paladar fue reportada de $11.65 \mathrm{~cm}$ (Otte y Venero, 1979), el cual se corresponde al presente valor promedio de $11.33 \mathrm{~cm}$.

Bustinza (2001) indica que no hay diferencia en el tamaño de la cabeza entre sexos y razas de alpaca. En el presente estudio se pudo comprobar la ausencia de diferencias 
estadísticas entre los valores relacionados al sexo.

Con respecto a los índices craneométricos, el índice facial es el único cuyo cálculo supera el $100 \%$ (104.84\%), pues la cara de la alpaca es muy angosta, de allí que la amplitud cigomática máxima sea mayor que la longitud víscero-craneal. Esto se presenta, asimismo, en el caso de los dromedarios cuyo índice facial es de $104.59 \%$ (Yahaya et al., 2011), a diferencia de otras especies como las ovejas Tuj y Morkaman cuyos índices son inferiores al 100\% (91.07 y 93.0\%, respectivamente)(Özcan et al., 2010), pero que igualmente son clasificadas como hiperleptenas (Krenzel, 2006).

En el caso de los camélidos, el triángulo facial está determinado por la bifurcación de la cresta sagital externa,siendo las medidas promedio del ancho, longitud y altura diferentes entre sí $(5.89,5.39$ y $5.08 \mathrm{~cm}$, respectivamente), lo que contradice los resultados de Adaro et al. (1992), quienes afirman que el triángulo facial de la alpaca es del tipo isósceles.

\section{Conclusiones}

- El cráneo de la alpaca, según las medidas e índices osteométricos obtenidos, no presenta dimorfismo sexual en el caso de animales adultos.

- La cabeza de alpaca adulta es del tipo dolicocéfalo, debido a que presenta un ICT igual a $46.43 \%$.

- La cara de alpaca adulta es de la clase hiperlepteno, debido a que presenta un IF igual a $103.17 \%$.

- El cráneo de la alpaca adulta es del tipo ultradolicocráneo, debido a que presenta un IC igual a $62.16 \%$.

- El índice mandibular es igual a $62.33 \%$.

- El triángulo facial de la alpaca adulta es del tipo escaleno.

\section{Literatura Citada}

1. Adaro L, Benavente M. 1992. Identificación de indicadores en el esqueleto axil de camélidos sudamericanos. Avances Cienc Vet 7(1). doi: 10.5354/07195273.1992.4679. [Internet]. Disponible en: http://www.revistas. uchile.cl/ index.php/ACV/article/view/4679/4566

2. Bustinza V. 2001. La alpaca. Conocimiento del gran potencial andino. Puno: UNA. $361 \mathrm{p}$.

3. [FAO] Organización de las Naciones Unidas para la Agricultura y la Alimentación. 2005. Situación actual de camélidos sudamericanos en Perú. Proyecto de Cooperación Técnica en apoyo a la crianza y aprovechamiento de los camélidos sudamericanos en la región andina. Roma: FAO. 63 p.

4. Fuentes L. 1953. Contribución a la osteología de la alpaca (Lama pacos). Tesis de bachiller. Lima: Univ Nacional Mayor de San Marcos. 69 p.

5. Galotta DR, Galotta JM. 1988. Osteología de la llama. Rev Cs Agr UCA 9(1): 19-61.

6. Guillén MJ, Quispe L, Baquerizo M. 2009. Índices cefálico total y corporal de la vicuña (Vicugna vicugna mensalis) adulta en el Centro de Investigación, Producción y Transferencia Tecnológica Tullpacancha - Huancavelica. Cienc Des 14(5): 51-55.

7. Krenzer U. 2006. Compendio de métodos antropológicos forenses para la reconstrucción del perfil osteo-biológico. Tomo 1. Guatemala: Serie de Antropología Forense. $33 \mathrm{p}$.

8. Louei A. 2013a. Anatomical study of the skull of the adult dogs and its clinical value during regional anesthesia. Global Vet 10: 459-463. doi: 10.5829/ idosi.gv.2013.10.4.7295

9. Louei A. 2013. Applied anatomy of the head regions of the one-humped camel (Camelus dromediarius) and its clinical implications during regional anesthesia. 
Global Vet 10: 322-326. doi: 10.5829/ idosi.gv.2013.10.3.72128

10. [ICVGAN] International Committee on Veterinary Gross Anatomical Nomenclature. 2012. [NAV]. Nómina Anatómica Veterinaria. $5^{\text {th }}$ ed. Hannover, Germany: ICVGAN. 177 p.

11. Navarrete M, Sato A. 2010. Aspectos anatómicos de la cría de alpaca. En: Sanidad de alpacas en la etapa neonatal. España: Ed Complutense. p 51-62.

12. Otte K, Venero J. 1979. Análisis de la craneometría diferencial entre la vicuña (Vicugna vicugna) y la alpaca (Lama guanicoe pacos). Stud Neotrop Fau E 14: $125-152$.

13. Özcan S, Aksoy G, Kürtül I, Aslan K, Özüdoðru Z. 2010. A comparative morphometric study on the skull of the Tuj and Morkaraman sheep. Kafkas Univ Vet Fak Derg 16: 111-114.

14. Pacheco V, Altamirano A, Guerra E. 1979. Guía de osteología de camélidos sudamericanos. Lima: Gabinete de Arqueología-Colegio Real, UNMSM. $16 \mathrm{p}$.

15. Quispe EC, Rodríguez TC, Iñiguez, Mueller JP. 2009. Producción de fibra de alpaca, llama, vicuña y guanaco en Sudamérica. Anim Genetic Res Inf 45: 1-14.
16. Romero M. 1989. Estudios biométricos y cálculos de correlación de la alpaca (Lama pacos), raza wacaya. Tesis de Médico Veterinario. Puno: UNA. 60 p.

17. Sañudo C. 2009. Valoración morfológica de los animales domésticos. España: Ed. Ministerio de Medio Ambiente y Medio Rural y Marino. $865 \mathrm{p}$.

18. Sisson S, Grossman JD. 2000. Anatomía de los animales domésticos. Tomo I y II. $5^{\mathrm{a}}$ ed. España: Ed Masson. 2302 p.

19. Yahaya A, Olopade JO, Kwari HD. 2011. Clinical Implications of craniometric indices of the one-humped camel (Camelus dromedarius) to oral health and clinical regional anaesthesia of the head. J Vet Anat 4: 19-31.

20. Yahaya A, Olopade JO, Kwari HD, Wiam IM. 2012. Investigation of the osteometry of the skull of the onehumped camels. Part II: sex dimorphism and geographical variations in adults. Ital J Anat Embryol 117: 34-44.

21. Zárate R. 2014. Caracterización anatómica de los senos paranasales de la alpaca (Vicugna pacos). Tesis de Médico Veterinario. Lima: Univ Nacional Mayor de San Marcos. 35 p.

22. Zhu L. 2012. Craniometrical studies on the skull of Tibetan gazelle (Procapra picticaudata). Int. J Morphol30): 196-198. 